\title{
Non-Destructive Cleaning of Carbon Nanotube Surfaces: Removal of Organic Contaminants and Chemical Residue with Oxygen Radicals
}

\author{
Mihail P. Petkov
}

Jet Propulsion Laboratory, California Inst. of Techn., 4800 Oak Grove Drive, Pasadena, CA 91109

Oxygen radicals (ORs) are highly efficient for in situ cleaning of organic contaminants from the interior of vacuum systems and sample surfaces [1]. ORs decompose organics into gasses $\left(\mathrm{H}_{2} \mathrm{O}, \mathrm{CO}\right.$, $\mathrm{CO}_{2}$ ), which are evacuated from the system. This phenomenon has been used to devise surface cleaning methods for a diverse range of materials. However, the application of OR cleaning to graphite and carbon nanotubes (CNTs) is not straightforward, as O plasma is known to ash both forms of carbon. This work [2] demonstrates a successful OR cleaning of surface organic contaminants (most likely hydrocarbons from the air), as well as chemical residue from the fabrication process, without inducing microstructural changes visible by SEM.

Using a commercial Evactron OR source, air was introduced into the system maintaining a constant pressure ( 0.56 torr), and ORs were produced from resonance RF excitation of $\mathrm{O}$ molecules. The $\mathrm{O}$ species were transported with the laminar flow, which is system-dependent. To roughly estimate a reasonable cleaning time, a fingerprint was deposited on a gold foil, and the disappearance of the $\mathrm{C}$ signature in EDS spectra was monitored as a function of exposure time (Fig. 1). Note that this is a severe contamination scenario, and such cleaning times are usually excessive. For the purpose of this work, C filament and CNT samples were treated for $100 \mathrm{~s}$ and $1000 \mathrm{~s}$. Figures 2-4 compare identical areas before (left images) and after $1000 \mathrm{~s}$ (right images).

Fig. 2 demonstrates the cleaning of organic surface contaminants, such as hydrocarbons from the air. Charged by the electron beam, they retain the imprint of the area scanned during prior SEM imaging. Three such typical rectangular shapes are visible in the "before" image (corners marked with arrows), and none are present in the "after" image. The charging at the edges of the C filaments in all "after" images is also reduced due to the removed hydrocarbon. Next, Fig. 3 shows the removal of chemical residue (dark spot) from the purification process. Although $1000 \mathrm{~s}$ were not sufficient to entirely eliminate the residue, the majority of it has been cleaned. For use in CNT fabrication, it is important to demonstrate that OR cleaning is non-destructive. To this end, a large number of high magnification SEM images were used (example: Fig. 4). None of them contained evidence of structural degradation, as that expected from energetic $\mathrm{O}$ plasma etching. This implies that OR cleaning appears to have no drawbacks relevant to CNT processing. Ongoing work is aimed at obtaining a conclusive confirmation by TEM and/or experiments with CNT devices.

In regard to $\mathrm{CNT}$ electronics, OR cleaning yields higher quality SEM images by removing surface contaminants. More importantly, the present results can have a significant impact on metal-to-CNT contact deposition processes, which are difficult to replicate for reasons that can be attributed to surface contaminants. Further investigation is in progress.

References:

[1] N. Sullivan et al, Microscopy and Microanalysis 8 (suppl. 2) (2002) 720CD

[2] This work was supported by the Jet Propulsion Laboratory, CIT, under a contract with NASA. 


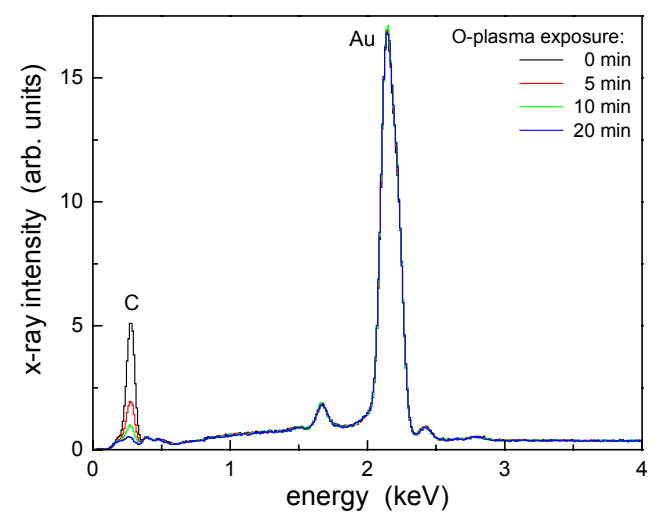

Fig. 1. Decomposition of a fingerprint on gold foil, demonstrated by the vanishing $\mathrm{C}$ signature in EDS spectra with OR exposure. These data are used to estimate the cleaning time for severely contaminated surfaces. All further experiments use 1000s OR exposure time.
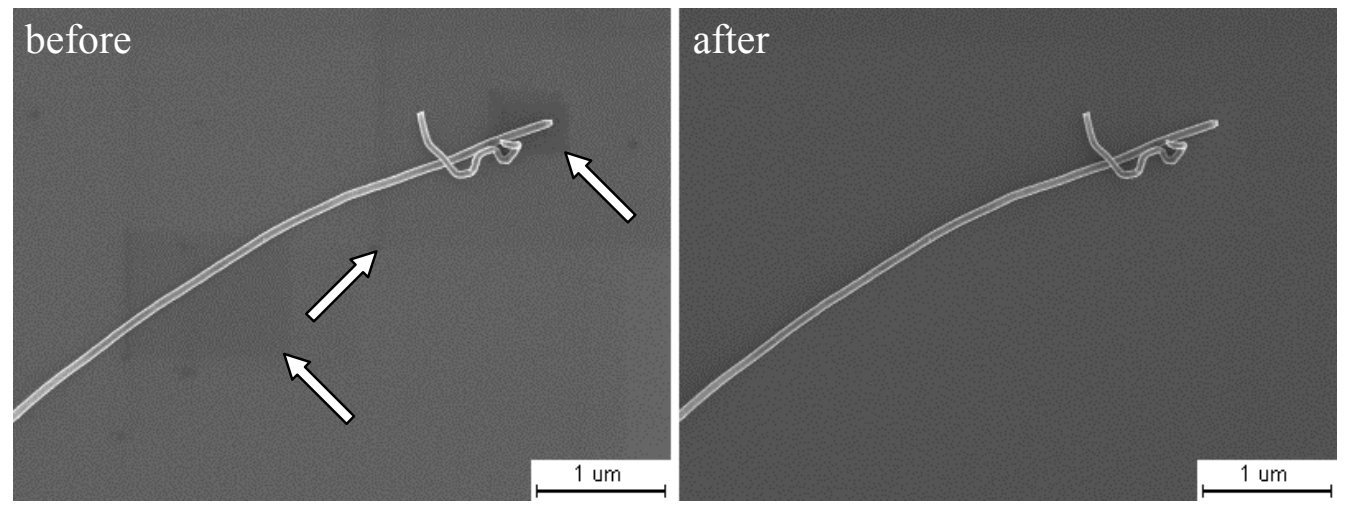

Fig. 2. Rectangular imprints of SEM scanned areas (left), usually due to charging of hydrocarbons on sample surfaces, are cleaned by OR radicals.
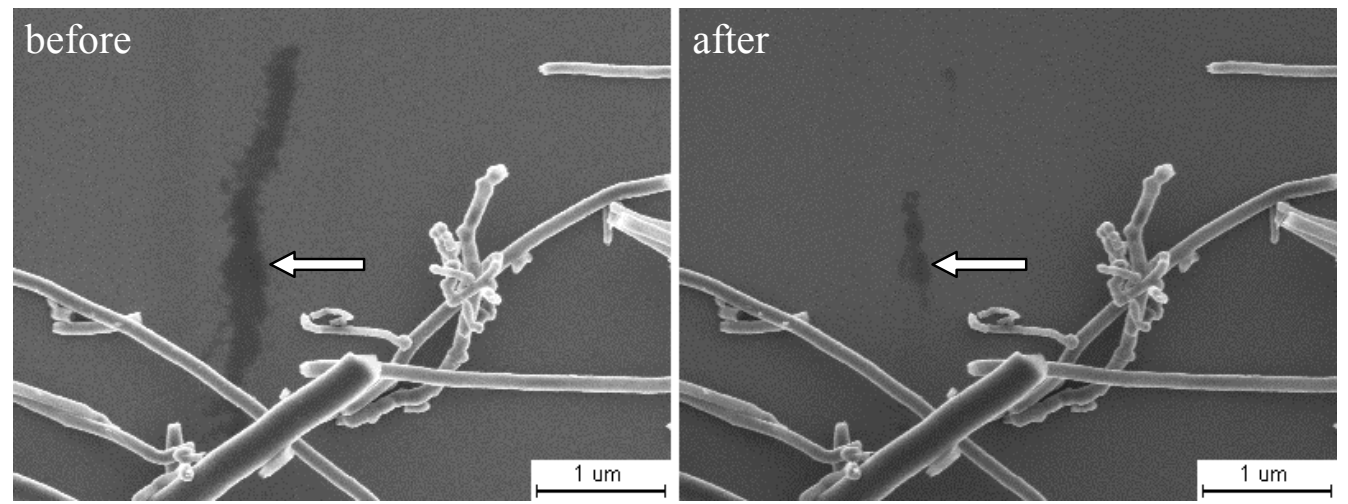

Fig. 3. Chemical residue from CNT fabrication is nearly removed.
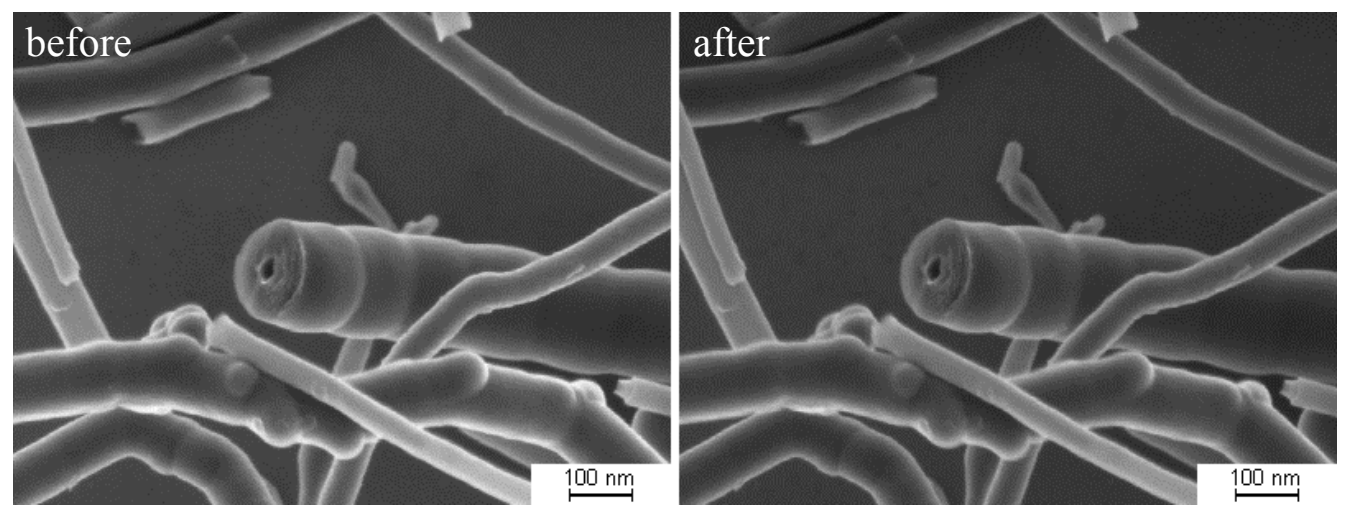

Fig. 4. High magnification SEM images show no OR-induced ill-effects, e.g., ashing. 